\title{
Characterization of Staphylococcus aureus Small Colony Variant (SCV) Clinical Isolates in Zaria, Nigeria
}

\author{
R. O. Ikala ${ }^{1 *}$, B. O. Olayinka ${ }^{1}$ and J. O. Ehinmidu ${ }^{1}$ \\ ${ }^{1}$ Department of Pharmaceutics and Pharmaceutical Microbiology, Ahmadu Bello University, Zaria, \\ Kaduna State, Nigeria.
}

Authors' contributions

This work was carried out in collaboration among all authors. Author ROI designed the study. Author $B O O$ performed the statistical analysis. Authors ROI and JOE wrote the protocol and wrote the first draft of the manuscript. Authors ROI and BOO managed the analyses of the study. Author JOE managed the literature searches. All authors read and approved the final manuscript.

Article Information

DOI: $10.9734 / A R R B / 2018 / v 30 i 630029$

Editor(s):

(1) Dr. Ibrahim Farah, Professor, Jackson State University, Mississippi, USA. (2) Dr. Gunanidhi Dhangadamajhi, Department of Biotechnology, North Orissa University, India. (3) Dr. Md. Torequl Islam, Nuclear of Pharmaceutical Technology (NTF), Postgraduate Program in Pharmaceutical Sciences, Federal University of Piaui, Brazil.

(4) Dr. George Perry, Dean and Professor of Biology, University of Texas at San Antonio, USA.

Reviewers:

(1) Abubakar Sunusi Adam, Kampala International University, School of Health Sciences, Uganda

(2) Osiyemi Joshua Adekunle, Olabisi Onabanjo University, Nigeria. Complete Peer review History: http://www.sdiarticle3.com/review-history/45466

Original Research Article

Received 17 December 2018

Accepted 19 February 2019

Published 14 March 2019

\section{ABSTRACT}

Staphylococcal isolates from specimen submitted to the Medical Microbiology laboratory of Ahmadu Bello University Teaching Hospital, Zaria were collected over a period of 6 months (February-July 2012), characterized by microbiological standard procedures and the $S$. aureus small colony variant (SCV) isolates were isolated. The antibiotic susceptibility pattern of the isolates was determined by the Kirby-Bauer-CLSI modified disc agar diffusion (DAD) technique. The SCV isolates were assessed for the carriage of four virulence genes; sdrE (putative adhesin) icaA (intracellular adhesin) hlg (hemolysin), Cna (collagen adhesin). A total of 258 non-duplicate staphylococcal isolates made up of $219(84 \%)$ S. aureus and $39(15 \%)$ coagulase-negative staphylococci (coNS) where obtained. A total of $48(22 \%)$ isolates were determined to be S. aureus SCV mainly from 
wound/abscess (31\%). S. aureus SCV isolates were generally resistant to all the nine antibiotics tested with only minimal sensitivity to tigecyclin $(10.4 \%)$ and ciprofloxacin (18.8\%). Statistically, there was no significant difference between the microbial load and the different antibiotics that were used, $(P \geq 0.05)$. None of the $S$. aureus SCV isolates carried the four virulence genes which were tested in this study. The results have therefore proved that $S$. aureus small colony variant exist in our environment and they are more resistant to most antimicrobial agent than their wild type.

Keywords: Staphylococcal; small colony variant; susceptibility; intracellular adhesion; collagen adhesion; hemolysin; putative adhesion; sensitivity.

\section{INTRODUCTION}

Staphylococcus aureus small colony variants (SCVs) are broadly defined as slow growing colonies with diameters roughly one tenth the parental strains when cultivated on agar plates [1]. Of particular importance are their decreased susceptibility to antibiotics and the absence of routine testing in clinical samples to detect their presence. These characteristics of SCV coupled with their capacity to revert to more rapidly growing form render them ideal candidates to provoke persistent human infections. Several decades of research now attest to their likely involvement in disease pathology $[2,3]$.

Staphylococcus aureus SCVs are generally reported to be auxotrophic for compounds that are biosynthesized into components of the electron transport system [4,5]. Menadione and hemin are the two most frequent substances that reverse the $S$. aureus SCV phenotype [6,7]. Reduced activity of the electron transport system can account for most of the features of the $S$. aureus SCVs. For example, a reduction in available ATP would slow growth, reduce pigment formation and decrease aminoglycoside transport.

Persistence and therapy refractory courses are characteristic features of $S$. aureus SCV infections which represent a serious difficulty in treating clinical cases $[1,8]$. In general, $S$. aureus SCV diseases show a wide variety of manifestations, ranging from superficial skin infection to life threatening conditions such as septicemia $[9,10]$. In particular, endovascular diseases such as endocarditis are frequently caused by $S$. aureus and in many clinical institutions $S$. aureus SCV has evolved as the leading pathogen of these infections [2].

S. aureus chronic and therapy refractory infections, as well as intracellular persistence have been associated with the SCV phenotypes [1]. However, because clinical SCVs are difficult to detect and are usually not stable but rapidly revert to their originally wild phenotype, the host cell response to SCVs is largely unknown [2]. When located intracellularly, SCVs has been reported to avoid activation of the host innate defense system and do not kill the host cells during persistence. This can be explained by the down regulation of important virulence factors in SCVs (e.g. $\alpha$ - toxin and proteases), which normally contribute to inflammation and tissue destruction [11].

In chronic infections, S. aureus SCV persists mainly intracellularly, where the bacteria are well protected against most antimicrobial treatments and against the host innate defense system [12]. There is even some preliminary evidence that the endothelial intracellular environment may favour the development of SCVs and bacterial regulatory processes due to non-protein coding RNAs and this might play a role in the formation of SCVs [9]. The intracellular SCVs contribute significantly to pathology and their reduced antibiotic susceptibility heralds a serious clinical problem.

S. aureus remain very versatile and exist almost everywhere including the hospital settings; therefore, this work aims at characterizing $S$. aureus small colony variant clinical isolates from Ahmadu Bello University Teaching Hospital, Zaria.

\section{MATERIALS AND METHODS}

\subsection{Culture Media}

Mannitol Salt Agar (MSA); Nutrient Agar (NA); Nutrient Broth (NB); Mueller-Hinton Agar; Blood Agar Base; all from Oxoid, UK.

\subsection{Antibiotic Discs}

The following antibiotic discs from Oxoid, UK were used; Gentamicin [10 $\mu \mathrm{g}$ ], ciprofloxacin [5 $\mu \mathrm{g}$, vancomycin [30 $\mu \mathrm{g}$ ] cefoxitin [30 $\mu \mathrm{g}$ ], erythromycin [15 $\mu \mathrm{g}]$ clindamycin [2 $\mu \mathrm{g}$, 
tigecycline $\left[\begin{array}{ll}15 & \mu \mathrm{g}\end{array}\right]$, cefuroxime $\left[\begin{array}{ll}30 & \mu \mathrm{g}\end{array}\right]$, amoxicillin $[30 \mu \mathrm{g}]$ representing the members of penicillin, third-generation cephalosporin, aminoglycoside, fluoroquinolone and glycopeptide classes.

\subsection{Collection of Clinical Isolates}

Suspected staphylococcal isolate from specimens submitted to the Medical Microbiology laboratory of ABUTH, Zaria were collected on NA slants over a period of 6 months. The slants were incubated for 18 hours at $37^{\circ} \mathrm{C}$ until there was visible growth. Slants were kept refrigerated until needed.

\subsection{Purification}

All cultures on NA slants were subcultured into nutrient broth, incubated overnight and the resulting cultures were streaked on nutrient agar plates and purified by single colony isolation.

\subsection{Preliminary Identification}

A loopful of overnight NB culture of the isolates was streaked on previously prepared Mannitol Salt Agar (MSA) plates. The plates were incubated at $37^{\circ} \mathrm{C}$ for $24 \mathrm{~h}$ under aerobic condition. After $24 \mathrm{~h}$ of incubation, the culture plates were examined recording the appearance, size, colour, and morphology of the colonies. Gram stain reaction, catalase test and coagulase test were carried out. Isolates that were grampositive cocci, catalase positive, and coagulated human plasma were considered $S$. aureus in this study.

\subsection{Isolation of Small Colony Variants}

\subsubsection{Growth on blood agar}

This was performed according to the method described by [12] Neut et al. (2003). A loopful of overnight nutrient broth cultures of confirmed $S$.aureus isolates were inoculated on a freshly prepared blood agar supplemented with $5 \%$ $\mathrm{NaCl}$. The cultured blood agar plates were incubated in an inverted position. The incubation lasted for $48-72$ hours at $37^{\circ} \mathrm{C}$. Isolates that yielded non pigmented and non-haemolytic pinpoint colonies were suspected to be small colony variants.

\subsubsection{Auxotrophy assay}

Auxotrophy was assayed by complementation with menadione sodium bisulphite (from Sigma-
Aldrich AB, Stockholm, Sweden). This was performed as a confirmatory test for SCVs using a five millimetre diameter filter paper discs (3MM paper Whatman International Maidstone, United Kingdom) which were soaked in menadione bisulphite solution at a concentration of 200 $\mu \mathrm{g} / \mathrm{ml}$ and aseptically placed with a forcep onto Mueller-Hinton plates inoculated with suspected $S$. aureus SCVs isolates.

Plates were incubated in inverted position aerobically for $24 \mathrm{hrs}$ at $37^{\circ} \mathrm{C}$. An increase in colony size proximal to the cellulose disc was interpreted as a positive result. This method was described by [6].

\subsection{Antibiotic Susceptibility Testing}

The antibiotic susceptibility pattern of the isolates was determined by the Kirby-Bauer-Clinical Laboratory Standards Institute (CLSI)-modified disc agar diffusion (DAD) technique. Discrete colonies of isolates on NA plate were emulsified in $3 \mathrm{ml}$ of PBS and the turbidity adjusted to 0.5 McFarland. Using sterile swab sticks, the surface of MHA was inoculated with the bacterial suspension; the antibiotic discs were aseptically applied to the surface of the inoculated agar plates. Within 30 minutes of applying the discs, the plates were inverted and incubated aerobically at $37^{\circ} \mathrm{C}$ for $16-18$ hours.

The diameter of the zones of growth inhibition were measured to the nearest millimeter and isolates classified as sensitive, intermediate or resistant based on CLSI interpretative chart of zone sizes [13].

\subsection{Molecular Identification of Virulence Genes}

\subsubsection{DNA isolation and purification}

The isolation and purification of genomic DNA from the isolates was done following miniprep method of [14] with modification.

\subsubsection{PCR amplification of virulence genes}

PCR amplification of four virulence genes was done as described by Peacock et al. [15]. Specific primer genes were used to amplify the genes. A $25 \mu \mathrm{l}$ of reaction mixture was made containing $20 \mu \mathrm{g}$ of template DNA, $100 \mu \mathrm{g}$ of primers, $160 \mathrm{Mm}$ of dNTP mix, $1.25 \mathrm{U}$ Taq polymerase, $1 \mathrm{x}$ Taq buffer and $0.5 \mathrm{Mm} \mathrm{MgCl}_{2}$. All the $S$. aureus SCV isolates were amplified 
Table 1. List of virulence genes and the primer sequences

\begin{tabular}{lll}
\hline Gene & Primer sequence & Amplicon size(bp) \\
\hline cna (collagen adhesin) & F: 5'TTCGTCACAATCAAGTTTGCC3' & 744 \\
& R: 3'CGGTGAAAAGTATGGGACG5' & \\
$h l g$ (hemolysin) & F:5'GCCAATCCGTTATTAGAAAATGC3' & 937 \\
& R: 3'CCATAGACGTAGCAACGGAT5' & \\
$i c a A($ intracellular adhesin) & F: 5'GATTATGTAATGTGCTTGGA3' & 770 \\
& R: 3'ACTACTGCTGCGTTAATAAT5' & \\
SdrE(putative adhesin) & F: 5'AGTAAAATGTGTCAAAAGA3' & 767 \\
& R: 3'TTGACTACCAGGCTATATC5' & \\
\hline
\end{tabular}

individually for four genes using the specific primers with 32 cycles of denaturation at $95^{\circ} \mathrm{C}$ for $1 \mathrm{~min}$, annealing at $50^{\circ} \mathrm{C}$ for icaA, $45^{\circ} \mathrm{C}$ for sdrE and $55^{\circ} \mathrm{C}$ for hlg and cna for $1 \mathrm{~min}$, extension at $72^{\circ} \mathrm{C}$ for 2 min on a thermocycler (PTC-100, MJ Research USA).

PCR products were resolved on $1.0 \%$ agarose gel at 60 volts for 2 hours. Gels were stained with ethidium bromide solution $(0.5 \mu \mathrm{g} / \mathrm{ml})$ and documentation was done using the Gel Doc system (Bio-Rad).

\subsection{Statistical Analysis}

One-way ANOVA was used to analyze results of the microbial load of the SCVs staphylococcus aureus and the different antibiotics used based on Tukey-Kramer's Multiple Comparisons Test using InStat3 Software, 2013 (significant at $\mathrm{P}<0.05)$.

\section{RESULTS}

A total of 258 staphylococcal isolates were obtained from clinical specimen submitted to the Medical Microbiology Laboratory of ABUTH, Zaria over the period of 6 months. A total of $48 / 219(22 \%)$ were determined to be S.aureus Small Colony Variants (SCV) phenotype. The distribution of SCVs by source shows that most of the isolates were from wound/abscess (31\%) as shown on Fig. 1.

From the antibiotic Susceptibility test, the zone of growth of inhibition obtained was classified based on the CLSI Interpretative chart of Antimicrobial Sensitivity Testing. Table 2 shows the outcome. Table 3 shows the antibiotic susceptibility pattern of the S.aureus wild type. Compared to the SCV, the wild type S. aureus was more susceptible to ciprofloxacin and gentamicin antimicrobial agents. The prevalent resistant phenotypes for both the wild type
S.aureus and the S.aureus SCV isolates where determined. Tables 4 and 5 show the outcome respectively. Fig. 2 shows the percentage resistance of $S$. aureus wild type and $S$. aureus SCV.

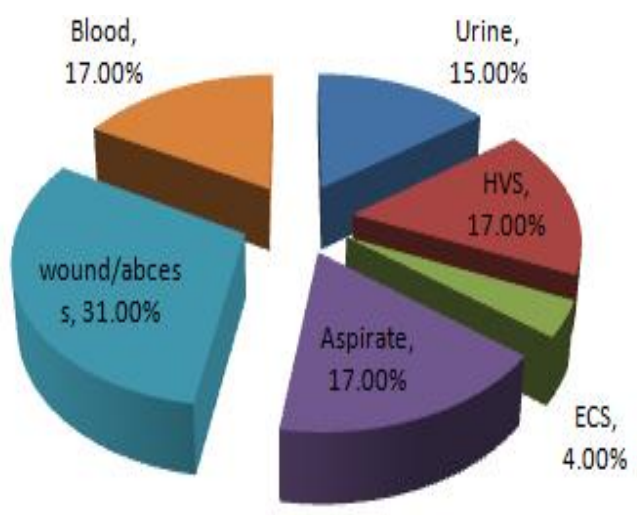

Fig. 1. Distribution of S.aureus SCV by specimen

\section{DISCUSSION}

The results from this work reveals that $S$. aureus small colony variant exist in our environment. The recovery rate of $S$. aureus small colony variants (SCVs) was $22 \%(48 / 219)$. The recovery rate in this study is in contrast to the report of [16]. He estimated the recovery rate of $S$. aureus SCVs in a general microbiology laboratory to be around $1 \%$. Another study by Becker et al. [17] reported the recovery rate of $S$. aureus SCVs to be 14 isolates in a period of 3 years.

Analysis of the distribution of the S. aureus SCVs by source showed that majority were from wound/abscess (31\%), blood (17\%), HVS (17\%), aspirate $(17 \%)$ and urine $(5 \%)$. The study of Gonzalez-Zorn and Courvalin [18] reported 5\% and $3 \%$ recovery rate from blood and wound respectively. 
Table 2. Susceptibility pattern of $S$. aureus Small Colony Variants isolates

\begin{tabular}{lllll}
\hline Antibiotics & Disc potency & Resistant (\%) & Intermediate (\%) & Sensitive (\%) \\
\hline Tigecycline & $15 \mu \mathrm{g}$ & 79.2 & 10.4 & 10.4 \\
Erythromycin & $15 \mu \mathrm{g}$ & 85.4 & 12.5 & 2.1 \\
Amoxicillin & $10 \mu \mathrm{g}$ & 100 & 0 & 0 \\
Cefuroxime & $30 \mu \mathrm{g}$ & 97.8 & 2.1 & 0 \\
Gentamicin & $10 \mu \mathrm{g}$ & 83.3 & 16.7 & 0 \\
Clindamycin & $2 \mu \mathrm{g}$ & 93.7 & 6.3 & 0 \\
Ciprofloxacin & $5 \mu \mathrm{g}$ & 81.3 & 0 & 18.8 \\
Cefoxitin & $30 \mu \mathrm{g}$ & 70.8 & 27.0 & 2.1 \\
Vancomycin & $30 \mu \mathrm{g}$ & - & - & 6.3 \\
\hline
\end{tabular}

Table 3. Antibiotic susceptibility pattern of wild type Staphylococcus aureus isolates

\begin{tabular}{lllll}
\hline Antibiotics & Disc potency & Resistant (\%) & Intermediate (\%) & Sensitive (\%) \\
\hline Tigecycline & $15 \mu \mathrm{g}$ & 19.3 & 12.3 & $\mathbf{6 8 . 4}$ \\
Erythromycin & $15 \mu \mathrm{g}$ & 55.6 & 19.9 & 24.6 \\
Amoxicillin & $10 \mu \mathrm{g}$ & 49.1 & 14.0 & 36.8 \\
Cefuroxime & $30 \mu \mathrm{g}$ & 72.5 & 15.8 & 11.7 \\
Gentamicin & $10 \mu \mathrm{g}$ & 17.5 & 8.2 & 74.3 \\
Clindamycin & $2 \mu \mathrm{g}$ & 59.1 & 9.4 & 31.6 \\
Ciprofloxacin & $5 \mu \mathrm{g}$ & 20.5 & 11.7 & 67.8 \\
Cefoxitin & $30 \mu \mathrm{g}$ & 44.5 & 26.4 & 29.2 \\
Vancomycin & 30 $\mathrm{\mu g}$ & - & - & 33.3 \\
\hline
\end{tabular}

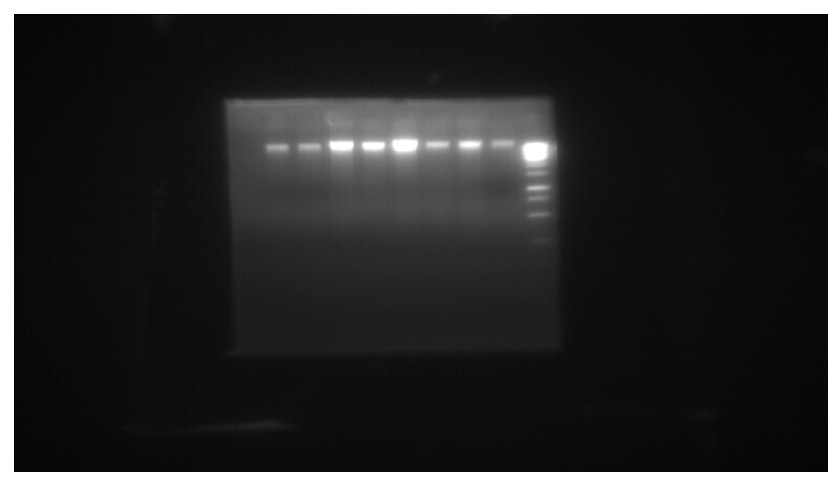

Fig. 2. Amplicon of the electrophoresis gel of the SCV Staphylococcus aureus

Table 4. Resistant phenotypes for wild type S. aureus isolates

\begin{tabular}{lll}
\hline Resistant phenotype & $\begin{array}{l}\text { Number of isolates } \\
(\mathbf{n}=\mathbf{1 7 1})\end{array}$ & Percentage of isolates (\%) \\
\hline E, AML, CXM, CN, CIP, VA,FOX, DA & 4 & 2 \\
AML, CXM, CN, DA, FOX, E, CIP & 4 & 2 \\
E, AML, CXM, CN, CIP, FOX & 18 & 11 \\
CIP, VA, DA, CXM, AML & 28 & 16 \\
CXM, AML, FOX, VA & 50 & 29 \\
TCG, CN, DA & 45 & 26 \\
DA, E & 11 & 6 \\
AML & 11 & 6 \\
\hline \multicolumn{2}{r}{ Key: TCG - Tigecycline, AML- Amoxicillin, DA - Clindamycin, E- Erythromycin, CXM - Cefuroxime, } \\
VA - Vancomycin, CN - Gentamicin, FOX - Cefoxitin, CIP - Ciprofloxacin
\end{tabular}


Table 5. Resistant phenotype of $S$. aureus SCV isolates

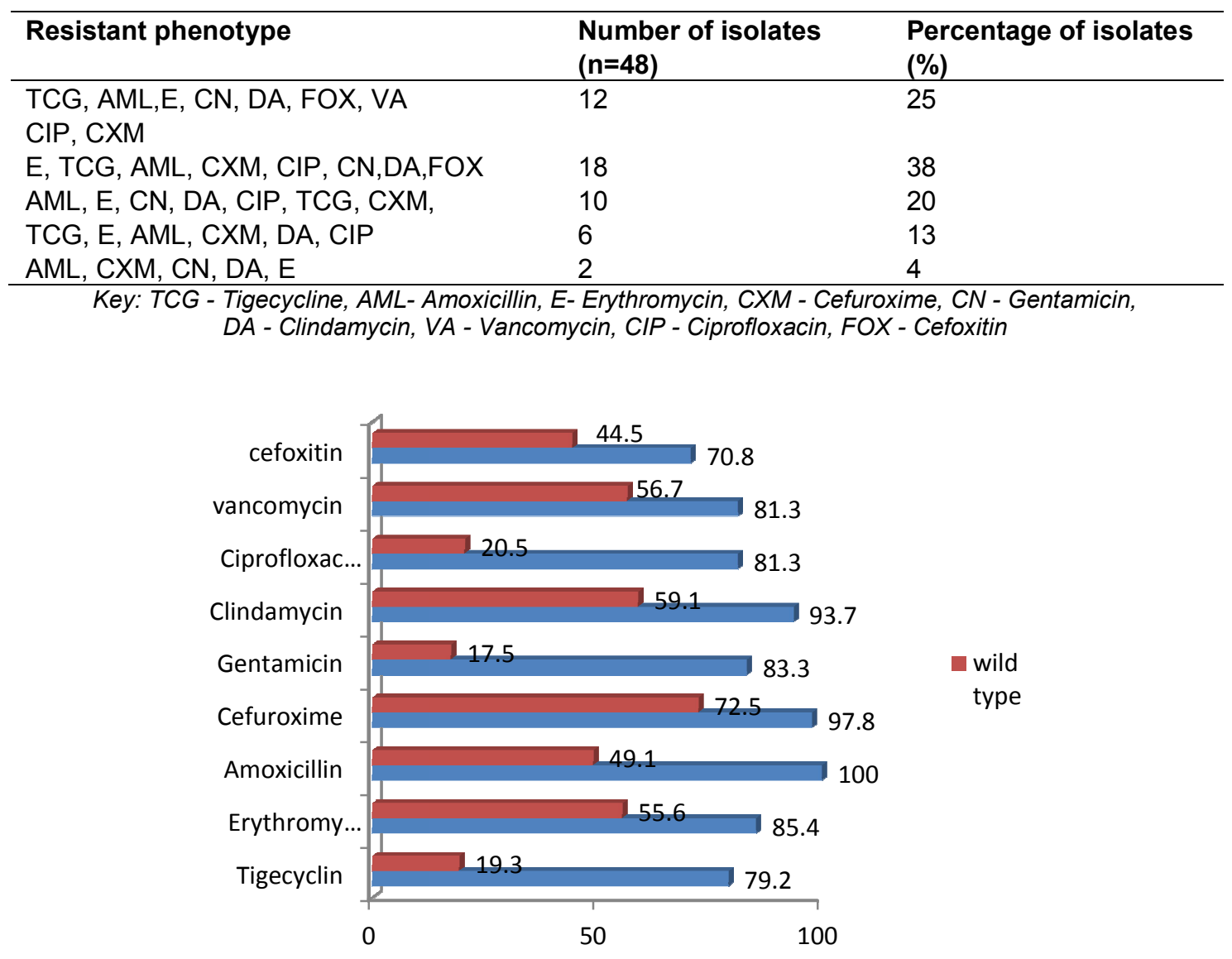

Fig. 3. Percentage resistance of S. aureus wild type and S. aureus SCV

Susceptibility testing of the small colony variant $S$. aureus isolates in this study against commonly available antibiotics showed that the isolates were generally resistant to $\beta$ - lactam drugs; (amoxicillin, cefuroxime), gentamicin, erythromycin and vancomycin with minimal sensitivity to tigecycline and ciprofloxacin antibacterial agent. The high level of resistance of the $S$. aureus small colony variants to most of these commonly available antibiotics used in this study is in agreement with the report of Proctor and Peters [19] who concluded that the depressed electron transport activity seen in auxotrophic SCVs may account for their in vitro resistance to a variety of antibiotics. In addition, the low content of ATP in SCVs causes inefficient transport of aminoglycoside into the cell, resulting in increased resistance to gentamicin and other aminoglycosides [19]. Moreover, the slow growth of SCVs and consequently cell wall division, reduces the effectiveness of antibiotics that act at the cell wall [20].

The susceptibility testing of the wild type $S$. aureus isolates in this study against the same antibiotics showed that the isolates were generally resistant to $\beta$ - lactam antibiotics (amoxicillin, cefuroxime), clindamycin, erythromycin and vancomycin while being generally sensitive to gentamicin (an aminoglycoside) and ciprofloxacin (a fluoroquinolone) antibacterial agents. In contrast to the result obtained in this study, [21] concluded that fluoroquinolones (e.g moxifloxacin) appeared consistently highly effective against the SCVs. Another study by Vaudaux et al. [22] reported that sensitivity to ciprofloxacin was higher for SCVs than for wild type $S$. aureus isolates with normal phenotype, while no remarkable difference was observed for other fluoroquinolones (moxifloxacin, levofloxacin and finafloxacin). 
The susceptibility level of the wild type $S$. aureus to ciprofloxacin is lower than the $99.7 \%$ reported by Akerele et al. [23]. This development may be connected with the increasing availability of the cheaper generics of fluoroquinolones in this environment leading to mis-use, over-use and gradual development of resistance.

From the determination of the virulence genes present in the S.aureus small colony variant isolates it was observed that none of the four virulent genes which were tested was present in the small colony variant isolates. This finding is in contrast to that reported previously by [24] who isolated SCVs that were thymidine auxotrophs and showed the over expression of intracellular adhesin. Further work is thus needed to determine how intracellular adhesin is activated in some types of clinical SCVs and not others. One possible explanation for the lack of detection of intracellular adhesin in the SCVs may be the kinetics of gene expression over time [25].

\section{CONCLUSION}

Clinical and laboratory findings lead to the conclusions that SCVs must be actively sought after in clinical microbiology, because they grow very slowly and can easily be missed. Particularly samples from individuals suffering from unusually persistent or recurrent infections should be examined meticulously for SCVs. In addition, it is most important to take SCVs into account as a possible cause of persistent infectious diseases when no bacteria or unusual microorganisms are found from such clinical specimen. Also due to reduced production of virulence factors by SCVs, they are adapted to the intracellular environment for long term persistence. An optimal treatment of SCV mediated infections has not been established but the $S$. aureus SCV in this study shows increased resistance to aminoglycosides and cell wall active antibiotics. Thus further study can be done in this field of study in order to understand the factors which select these phenotypes in the host and the genetic basis of this type of auxotrophy (menadione auxotrophy).

\section{ETHICAL APPROVAL}

As per university standard guideline participant ethical approval has been collected and preserved by the authors.

\section{DISCLAIMER}

This manuscript title was presented in the conference.

Conference name: 13th National Conference and Scientific Meeting, Nigeria Association of Pharmacists in Academia, Faculty of Pharmacy, University of Ibadan.

Available:https://www.researchgate.net/profile/Bu sayo_Olayinka/publication/284028491_Character ization_of_Staphylococcus_aureus_Small_Colon y Variant SCV Clinical Isolates from Ahmadu Bello University Teaching Hospital Žaria/links /564b246008ae127ff9880538/Characterizationof-Staphylococcus-aureus-Small-Colony-VariantSCV-Clinical-Isolates-from-Ahmadu-BelloUniversity-Teaching-Hospital-Zaria.pdf 10th - 14th August, 2015.

\section{COMPETING INTERESTS}

Authors have declared that no competing interests exist.

\section{REFERENCES}

1. Proctor RA, Von Eiff C, Kahl BC, Becker K, Mcnamara $P$, Herrmann $M$, Peters $G$. Small colony variants: A pathogenic form of bacteria that facilitates persistent and recurrent infections. Nat. Rev. Microbiol. 2006;4:295-30.

2. Sendi P, Proctor RA. Staphylococcus aureus as an intracellular pathogen: The role of small colony variants. Trends Microbiol. 2009;17:54-58.

3. Kim NH, Kang YM, Han WD, Park KH, Yoo JI, Lee DG, Park C, Song KH, Kim ES, Park SW, Kim NJ, Oh MD, Kim HB. Small-colony Staphylococcus aureus bacteremia. Microb. Drug Resist. 2016;22:538-544.

4. Von Eiff C, Winkelmann W, Peters G. Recovery of small colony variants of Staphylococcus aureus following gentamicin bead placement for osteomyelitis. Clin. Infect. Dis. 1997;25: 1250-1251.

5. Patrizia M, Alessandra DA, Graziona M, Anna M, Robarto B, Marta B, Raffaele L, Laura Minicucci. Characterization of Staphylococcus aureus small colony variant strains isolated from Italian patients attending a regional cystic fibrosis care centre. New Microbiology. 2015;38:235243. 
6. Lannergard J, von Eiff C, Sander G, Cordes T, Seggewiss J, Peters G, Proctor RA, Becker K, Hughes D. Identification of the genetic basis for clinical menadione-auxotrophic smallcolony variant isolates of $S$. aureus. Antimicrob. Agents Chemother. 2008;52: 4017-4022.

7. Mellissa AD, Randall JO, Wesley SI, Adiana ER, James MM. Identification of point mutations in clinical Staphylococcus aureus strains that produce small variants auxotrophic for menadione. Infect. Immun. 2014;82(4):1600-1605.

8. Proctor RA, Peters G. Small colony variants in staphylococcal infections: Diagnostic and therapeutic implications. Clin. Infect. Dis. 2008;27:419-422.

9. Lowy FD. Staphylococcus aureus infections. New England Journal of Medicine. 1998;339:520-532.

10. Suwantarat N, Rubin M, Bran L, Tekle T, Boyle MP, Caroll KC, Jennings MT. Frequency of small colony variants and antimicrobial susceptibility of methicillin resistant Staphylococcu aureus in cystic fibrosis patients. Diagn. Microbiol. Infect. Dis. 2018;90(4):296-299.

11. Haslinger-Loffler B, Menzies BE, Peterson ML. Multiple virulence factors are required for Staphylococcus aureus- induced apoptosis in Endothelial cells. Cell Microbiol. 2005;7:1087-1097.

12. Norville P. Small colony variants in Staphylococcus aureus and other species: Antibiotic selection, antimicrobial susceptibility and biofilm formation. Ph.D Thesis, Cardiff University; 2011.

13. Cheesbrough M. District laboratory practice in tropical countries. Part 2. Cambridge University press UK. 2002;136143.

14. Ausbel FM, Brent R, Kingston RE, Moore DD, Seidman JG, Smith JA. Miniprep of bacterial genomic DNA. Short protocols in molecular biology $4^{\text {th }}$ ed. USA: John Willey and Sons. 2004;2:12-3.

15. Peacock SJ, Moore CE, Justice A, Kantzanon M, Story L, Mackie K. Virulent combination of adhesion and toxin genes in natural populations of Staphylococcus aureus. Infect Immun. 2002;70:4987-96.

16. Acar JF, Goldstein FW, Lagrange $P$. Human infections caused by thiamine- or menadione-requiring Staphylococcus aureus. J. Clin. Microbiol. 1998;8:142147.

17. Becker K, Laham NA, Fegeler W, Proctor RA, Peters G, Von Eiff C. Fouriertransform infrared spectroscopic analysis is a powerful tool for studying the dynamic changes in Staphylococcus aureus smallcolony variants. J. Clin. Microbiol. 2006;44: 3274-3278.

18. Gonzalez-Zorn B, Courvalin P. vanAmediated high level glycopeptide resistance in MRSA. Lancet Infectious Diseases. 2003;3:67-68.

19. Proctor RA, Peters G. Small colony variants in staphylococcal infections: Diagnostic and therapeutic implications. Clin. Infect. Dis. 1998;27:419-422.

20. Looney D, MacDougall C, Powell JP, Johnson CK. Hospital and community fluoroquinolone use and resistance in Staphlococcus aureus and Escherichia coli in 17 US hospitals. Clinical Infectious Diseease. 2000;41:435440.

21. Nguyen HA, Denis $O$, Vergison $A$, Theunis A, Tulkens PM, Struelens MJ, Van Bambeke F. Intracellular activity of antibiotics in a model of human THP macrophages infected by a Staphylococcus aureus small-colony variant strain isolated from a cystic fibrosis patient: Pharmacodynamic evaluation. Science. 2009;34:234-240.

22. Vaudaux P, Mcnamara $P$, Herrmann M, Peters G. Increased expression of clumping factor and fibronectin-binding proteins by hemB mutants of Staphylococcus aureus expressing small colony variant phenotypes. Infect. Immun. 2011;70:5428-5437.

23. Akerele J, Abdulimen $\mathrm{P}$, Okonfua $\mathrm{F}$. Prevalence of asymptomatic bacteriuria among pregnant women in Benin City, Nigeria. J. Obtet Gynaecol. 2001;21(2): 141-144.

24. Kahl B, Duebbers A, Lubritz G, Haeberle J, Koch HG, Ritzerfeld B, Reilly M, Harms E, Proctor RA, Herrmann M, Peters $G$. Population dynamics of persistent Staphylococcus aureus isolated from the airways of cystic fibrosis patients during a 6-year prospective study. J. Clin. Microbiol. 2003;41:44244427. 
25. Kipp F, Jorgensen JH, Ferraro MJ. Detection of Staphylococcus aureus by $16 S$ rRNA directed in situ hybridisation in a patient with a brain abscess caused by small colony variants. J. Neurol. Neurosurg. Psychiatry. 2003;74:1000-1002.

(c) 2018 Ikala et al.; This is an Open Access article distributed under the terms of the Creative Commons Attribution License (http://creativecommons.org/licenses/by/4.0), which permits unrestricted use, distribution, and reproduction in any medium, provided the original work is properly cited.

Peer-review history:

The peer review history for this paper can be accessed here: http://www.sdiarticle3.com/review-history/45466 